\title{
DNA Cytometry for Differentiation Between Low- and Medium- grade Dysplasia in Intraductal Papillary Mucinous Neoplasms
}

\author{
FRITZ KLEIN $^{1 *}$, TIMM DENECKE ${ }^{2 *}$, WLADIMIR FABER ${ }^{1}$, CHRISTIAN JÜRGENSEN $^{3}$, \\ ANJA SCHIRMEIER ${ }^{1}$, HUSSEIN AL-ABADI ${ }^{1}$, THULA C. WALTER ${ }^{2}$, NADINE AL-ABADI $^{1}$, \\ THOMAS MALINKA ${ }^{1}$, JOHANN PRATSCHKE ${ }^{1}$ and MARCUS BAHRA ${ }^{1}$ \\ Departments of ${ }^{1}$ Surgery, ${ }^{2}$ Diagnostic and Interventional Radiology, and ${ }^{3}$ Gastroenterology, \\ Infectious Diseases and Rheumatology, Campus Mitte, Charite University of Medicine, Berlin, Germany
}

\begin{abstract}
Background/Aim: The indication for resection of cystic pancreatic lesions is usually performed by sectional imaging criteria, such as the Sendai criteria. The aim of this study was to analyze a possible correlation between DNA cytometry and Sendai criteria for the differentiation between low-grade intraductal papillary mucinous neoplasms (IPMNA) and medium-grade dysplasia (IPMN-B). Materials and Methods: Histopathological analysis, DNA index and preoperative Sendai criteria were determined in 16 patients who underwent pancreatic resection for IPMN. Results: All patients with IPMN-B showed aneuploid histograms with $D N A$ indices $\geq 1.3$, whereas three out of four patients with $I P M N-A$ had diploid DNA indices $\leq 1.3$. All 11 patients with one or more high-risk stigmata and aneuploid histograms had IPMN-Bs, whereas both patients who were Sendainegative and diploid in the DNA analysis had an IPMN-A. Conclusion: DNA index may be an important diagnostic tool for the differentiation of different IPMN types beyond the traditional Sendai criteria.
\end{abstract}

Pancreatic intraductal papillary mucinous neoplasia (IPMN) is slow-growing mucin-producing cell dysplasia of the ductal epithelium which causes a dilation of the ducts filled with mucin of high viscosity becoming visible as a cystic dilation of the involved duct segments. Non-invasive IPMNs can be differentiated into low-grade dysplasia (IPMN-A, formerly adenoma), medium-grade dysplasia (IPMN-B, formerly

*These Authors contributed equally to this study.

Correspondence to: Dr. med. Fritz Klein, Department of Surgery, Charité-Universitätsmedizin, Augustenburger Platz 1, 13353 Berlin, Germany. Tel: +49 30652033, Fax: +49 30450552900, e-mail: fritz.klein@charite.de

Key Words: IPMN, cystic neoplasia, pancreas, DNA index, Sendai criteria. borderline dysplasia) and carcinoma in situ (high-grade dysplasia, IPMN-C). The progression of IPMN into an adenoma-carcinoma sequence makes the lesion a precursor of invasive ductal adenocarcinoma (1). The actual malignant potential and duration of dedifferentiation of IPMN is not definitively known and varies individually (2). This and the high prevalence of pancreatic IPMN leaves us with a dilemma as pancreatic surgery is still of high risk and should thus be performed only in those patients who really benefit from resection.

In order to solve this dilemma, non-invasive imaging plays a key role for the first assessment of IPMN. Several features which serve as risk stratification parameters were defined at the 2006 consensus conference in Sendai and updated in 2012 $(3,4)$. These parameters include cyst size of $>3 \mathrm{~cm}$, thickened enhanced cyst walls, non-enhanced mural nodules, and main pancreatic duct size of $>10 \mathrm{~mm}$ (4). However, these criteria can only serve as surrogate parameters as the invasive partition of the tumor may be small and the dysplastic epithelium becomes visible only indirectly because of the secreted mucin causing duct dilation. While the risk of malignant transformation increases with positivity of Sendai criteria, the predictive value of bearing carcinoma in situ is rather low and, on the other hand, even in Sendai-negative IPMN lesions, carcinoma can sometimes be found upon resection. Especially in the differentiation of low- and intermediate-grade dysplastic IPMN, the imaging surrogate parameters derived from computed tomography (CT) and magnetic resonance imaging (MRI) are not sufficiently accurate (5). This is why endoscopic ultrasound-guided fineneedle aspiration (EUS-FNA) is employed to harvest lesion content and to analyze it for tumor markers and cytology. However, even this is not highly specific and additional parameters acquired from FNA samples are needed to improve objective criteria for surgical decision-making.

The DNA index and DNA ploidy status have been reported to serve as prognostic and predictive biomarkers of cancer for many different types of malignant tumors (6-10). Weger et al . 
(11), Porschen et al. (12) and Klein et al. (13) observed a strong correlation between DNA ploidy status and survival of patients with pancreatic cancer. In a prospective clinical trial, Kamphues et al. showed that the DNA index serves as an independent predictive marker in patients with pancreatic head cancer (14). In another prospective clinical trial, the same research group demonstrated that the DNA index is also an independent predictive marker of intrahepatic cellular carcinoma (15). With the perspective of using the DNA index for FNA samples in patients with IPMN, the aim of the present study was to investigate the potential of this cytological marker for further discrimination of low- and intermediate-grade dysplasia in resected lesions in order possibly to identify a reliable reference standard for further determination of the index in this clinical constellation. In addition, our results of the DNA Index analysis were compared to the presence of worrisome features according to the Sendai criteria in the preoperative imaging findings in an attempt to analyze a relevant clinical correlation in the preoperative interdisciplinary diagnostic work-up.

\section{Materials and Methods}

Patients. A total of 16 patients who underwent pancreatic resection at our Institution for benign IPMN (low- or medium-grade dysplasia) for which the malignant potential could not be established preoperatively and for whom preoperative crosssectional imaging (CT w/wo magnetic resonance imaging MRI), as well as sufficient material of the resected specimen for DNA index determination were available, were enrolled retrospectively. The detailed preoperative data are summarized in Table I. No significant differences were found regarding age $(p=1.0)$, body mass index (BMI, $p=0.26$ ) and American Society of Anesthesiologists classification $(p=0.41)$. No differences concerning alcohol and nicotine anamnesis were recorded $(p=0.55$ and $p=0.39$, respectively). This study was performed in accordance with the Declaration of Helsinki and its amendments and approved by the Institutional Ethic Committee (approval number: EA2/035/14).

Radiologic imaging. All patients underwent multiphasic contrastenhanced CT with/without MRI (including MR-cholangiopancreatography). The imaging material was reviewed by two experienced abdominal radiologists in consensus who were blinded to the clinical data. High-risk stigmata (main duct diameter $>10 \mathrm{~mm}$, lesion size $>3 \mathrm{~cm}$, thickened enhanced cyst walls and non-enhanced mural nodules) were documented in accordance with the 2012 International Consensus Guidelines (4).

Surgery. The type of pancreatic resection was defined by the size and location of the tumor. The abdomen was systematically examined intraoperatively, including intraoperative ultrasound of the pancreatic gland in all patients.

Histopathology and DNA index. The tumor specimen was divided into two parts after resection. One part was analyzed using a standard histopathological examination. The other part was frozen and preserved at $-80^{\circ} \mathrm{C}$. The preparation of monolayer smears was performed using the cell separation technique $(16,17)$. The staining of monolayer smears was performed using the method reported by Feulgen (17). For each slide, 900 tumor cells were analyzed, and the mean nuclear area and the DNA content were recorded. For DNA image cytometry, a computer-assisted video image analysis system (Cydok from Hilgers, Königswinter, Germany; and CM-1 from Hund, Wetzlar, Germany) combined with a conventional light microscope was used. In each case, the mean DNA content of 30 leukocytes was calculated and served as an internal standard for the diploid DNA value.

The samples were only included when the variation in DNA content differed by less than $3 \%$ from that of the reference cell population. Documentation of the results was performed with a DNA histogram with a class width of $0.1 \mathrm{c}$. The histograms were analyzed according to the recommendations of the Consensus Report of the European Society for Analytical Cellular Pathology (18). In order to calculate the DNA index, the mean nuclear DNA content of the $\mathrm{G}_{0} / \mathrm{G}_{1}$ compartment of the neoplastic cell population was divided by the DNA content of the $G_{0} / G_{1}$ compartment of a similarly processed group of known diploid reference cells. Finally, the results of the histological examination were correlated with the findings of DNA cytometry.

Statistical analysis. Quantitative variables are expressed as medians and ranges. The differences between the DNA indices of histopathological groups were analyzed using a $t$-test. All statistical tests were performed with SPSS 19.0 statistical software program (IBM Corp., Armonk, NY, USA)

\section{Results}

Procedural data and clinical course. The detailed operative data from both groups are summarized in Table II. No significant differences were found regarding the type $(p=0.54)$ or duration $(p=0.78)$ of operation. The intraoperative need for packed red blood cell units $(p=0.39)$ and fresh-frozen plasma units $(p=0.61)$ did not differ significantly between groups, and postoperative complications were similar in both groups $(p=0.41)$. The in-hospital stay $(p=0.95)$ and intensive-care unit stay $(p=1.0)$ were also similar. No intraoperative death was recorded. However, one patient with IPMN-A died during their hospital stay due to acute bleeding of a pancreatic fistula.

Imaging criteria. The detailed radiological and pathological data are summarized in Table III. In the group with adenoma (IPMN-A), only one patient with branch-duct (BD)-IPMN was positive for the Sendai criteria. None of the other adenomas [two BD-IPNMs and one main-duct (MD)-IPMN] fulfilled the Sendai criteria. In the group with borderline tumors (IPMN-B), we observed a heterogeneous distribution of the Sendai criteria. Overall (cumulative for MD-IPMN and BD-IPMN), one patient in this group did not fulfill any Sendai criteria; seven patients fulfilled one criterion; three patients fulfilled two criteria; and one patient fulfilled three Sendai criteria. In the subgroup with BD-IPMN histological type IPMN-B ( $n=5$ patients), the distribution of Sendai criteria was also heterogeneous. One patient did not fulfill 
Table I. Study population

\begin{tabular}{|c|c|c|c|c|c|c|c|}
\hline Patient no. & Histology & Gender & $\begin{array}{l}\text { Patient age } \\
\text { (years) }\end{array}$ & $\begin{array}{c}\text { ASA } \\
\text { classification }\end{array}$ & $\begin{array}{c}\mathrm{BMI} \\
\left(\mathrm{kg} / \mathrm{m}^{2}\right)\end{array}$ & $\begin{array}{l}\text { Positive nicotine } \\
\text { anamnesis }\end{array}$ & $\begin{array}{l}\text { Positive alcohol } \\
\text { anamnesis }\end{array}$ \\
\hline 1 & IPMN-A & Male & 72 & II & 22.1 & No & No \\
\hline 2 & IPMN-A & Female & 87 & II & 21.5 & No & No \\
\hline 3 & IPMN-A & Male & 57 & II & 24.5 & No & No \\
\hline 4 & IPMN-A & Female & 40 & II & 18.8 & No & No \\
\hline 5 & IPMN-B & Male & 67 & III & 33.5 & No & No \\
\hline 6 & IPMN-B & Female & 70 & II & 23.2 & No & No \\
\hline 7 & IPMN-B & Male & 72 & III & 20.5 & No & No \\
\hline 8 & IPMN-B & Female & 47 & II & 26.5 & Yes & No \\
\hline 9 & IPMN-B & Female & 56 & II & 27.9 & No & No \\
\hline 10 & IPMN-B & Female & 72 & II & 19.5 & No & No \\
\hline 11 & IPMN-B & Male & 63 & II & 22.3 & Yes & No \\
\hline 12 & IPMN-B & Male & 58 & II & 29.6 & Yes & Yes \\
\hline 13 & IPMN-B & Male & 73 & III & 22.7 & No & No \\
\hline 14 & IPMN-B & Female & 76 & II & 20.8 & No & No \\
\hline 15 & IPMN-B & Female & 66 & II & 23.0 & No & Yes \\
\hline \multirow[t]{2}{*}{16} & IPMN-B & Male & 49 & I & 24.1 & No & No \\
\hline & p Value* & 0.72 & 1.0 & 0.41 & 0.26 & 0.39 & 0.55 \\
\hline
\end{tabular}

ASA: American Society of Anesthesiologists; BMI: body mass index; IPMN-A: low-grade dysplasia; IPMN-B: medium-grade dysplasia. *Comparison between IPMN-A and IPMN-B.

Table II. Procedural data and clinical course of patients in this study.

\begin{tabular}{|c|c|c|c|c|c|c|c|c|}
\hline $\begin{array}{l}\text { Patient } \\
\text { no. }\end{array}$ & $\begin{array}{l}\text { Histological } \\
\text { type }\end{array}$ & $\begin{array}{l}\text { OP } \\
\text { type }\end{array}$ & $\begin{array}{l}\text { In-hospital } \\
\text { stay (days) }\end{array}$ & $\begin{array}{l}\text { ICU stay } \\
\text { (days) }\end{array}$ & $\begin{array}{c}\text { Duration of } \\
\text { operation (min) }\end{array}$ & $\begin{array}{c}\text { Blood intraoperative } \\
\text { need }\end{array}$ & $\begin{array}{l}\text { Fresh frozen plasma } \\
\text { intraoperative need }\end{array}$ & $\begin{array}{l}\text { Clavien grade } \\
\text { postoperative }\end{array}$ \\
\hline 1 & IPMN-A & PPPD (PG) & 30 & 5 & 320 & No & Yes & None \\
\hline 2 & IPMN-A & PPPD (PG) & 19 & 6 & 275 & No & No & II \\
\hline 3 & IPMN-A & PPPD (PG) & 15 & 0 & 415 & No & No & $\mathrm{V}$ \\
\hline 4 & IPMN-A & PPPD (PG) & 15 & 0 & 272 & No & No & None \\
\hline 5 & IPMN-B & PPPD (PG) & 17 & 2 & 414 & Yes & No & IIIB \\
\hline 6 & IPMN-B & PPPD (PG) & 19 & 3 & 256 & No & No & II \\
\hline 7 & IPMN-B & PPPD (PG) & 21 & 2 & 340 & Yes & Yes & IIIa \\
\hline 8 & IPMN-B & PPPD (PG) & 16 & 1 & 322 & No & No & None \\
\hline 9 & IPMN-B & DP & 11 & 1 & 150 & No & No & None \\
\hline 10 & IPMN-B & PPPD (PG) & 15 & 0 & 350 & No & No & None \\
\hline 11 & IPMN-B & $\mathrm{DP}$ & 9 & 1 & 211 & No & No & None \\
\hline 12 & IPMN-B & PPPD (PG) & 18 & 0 & 337 & No & No & None \\
\hline 13 & IPMN-B & Pancreatectomy & 24 & 11 & 353 & Yes & Yes & II \\
\hline 14 & IPMN-B & PPPD (PG) & 27 & 1 & 369 & No & No & IIIB \\
\hline 15 & IPMN-B & PPPD (PG) & 17 & 1 & 357 & No & No & None \\
\hline \multirow[t]{2}{*}{16} & IPMN-B & PPPD (PG) & 20 & 1 & 322 & No & No & II \\
\hline & $p$-Value* & 0.54 & 0.95 & 1.0 & 0.78 & 0.39 & 0.61 & 0.41 \\
\hline
\end{tabular}

IPMN-A: Low-grade dysplasia; IPMN-B: medium-grade dysplasia; PPPD (PG): pancreaticoduodenectomy (pancreaticogastrostomy); DP: distal pancreatectomy; ICU: intensive care unit. *Comparison between IPMN-A and IPMN-B.

any Sendai criteria; two fulfilled one; one patient fulfilled two; and one patient met three of the criteria for malignancy. Considering only the BD-IPMN type, no significant difference between the IPMN-A and IPMN-B groups was found $(p=0.29)$. Overall, 11 out of 12 patients in the IPMN-
B group met at least one positive high-risk Sendai criterion. DNA index. All patients with IPMN-B presented aneuploid histograms with a median DNA index $\geq 1.3$ (range=1.3-1.5). In contrast, in the IPMN-A group, diploid histograms with a median DNA index $\leq 1.3$ (range $=1.0-1.3$ ) were observed in 
Table III. Histological and radiological data of patients in this study.

\begin{tabular}{lcccccccccc}
\hline $\begin{array}{l}\text { Patient } \\
\text { no. }\end{array}$ & $\begin{array}{c}\text { Histology } \\
\text { type }\end{array}$ & $\begin{array}{c}\text { Tumor } \\
\text { type }\end{array}$ & DNA & $\begin{array}{c}\text { Ploidy } \\
\text { Index }\end{array}$ & $\begin{array}{c}\text { Duct width } \\
(\mathrm{mm})\end{array}$ & $\begin{array}{c}\text { Duct width } \\
>9 \mathrm{~mm}\end{array}$ & $\begin{array}{c}\text { Max. diameter } \\
(\mathrm{cm}, \mathrm{axial})\end{array}$ & $\begin{array}{c}\text { Size } \\
>3 \mathrm{~cm}\end{array}$ & $\begin{array}{c}\text { Mural } \\
\text { nodules }\end{array}$ & $\begin{array}{c}\text { Number of positive } \\
\text { Sendai criteria }\end{array}$ \\
\hline 1 & IPMN-A & Branch & Diploid & 1.0 & 4 & No & 3.8 & Yes & Yes & 2 \\
2 & IPMN-A & Main & Aneuploid & 1.3 & 3 & No & 2.2 & No & No & 0 \\
3 & IPMN-A & Branch & Diploid & 1.1 & 1 & No & 2.0 & No & No & 0 \\
4 & IPMN-A & Branch & Diploid & 1.2 & 2 & No & 1.5 & No & No & 0 \\
5 & IPMN-B & Branch & Aneuploid & 1.3 & 9 & No & 2.2 & No & No & 0 \\
6 & IPMN-B & Branch & Aneuploid & 1.5 & 1.5 & No & 2.7 & No & Yes & 1 \\
7 & IPMN-B & Main & Aneuploid & 1.3 & 11 & Yes & 2.4 & No & No & 1 \\
8 & IPMN-B & Main & Aneuploid & 1.4 & 5 & No & 3.5 & Yes & No & 1 \\
9 & IPMN-B & Main & Aneuploid & 1.3 & 1 & No & 2.8 & No & Yes & 1 \\
10 & IPMN-B & Branch & Aneuploid & 1.3 & 5 & No & 2.0 & No & Yes & 1 \\
11 & IPMN-B & Main & Aneuploid & 1.4 & 3 & No & 2.6 & No & Yes & 1 \\
12 & IPMN-B & Main & Aneuploid & 1.3 & 8 & No & 4.3 & Yes & No & 1 \\
13 & IPMN-B & Main & Aneuploid & 1.3 & 5.6 & No & 12.0 & Yes & Yes & 2 \\
14 & IPMN-B & Main & Aneuploid & 1.4 & 3.7 & No & 3.5 & Yes & Yes & 2 \\
15 & IPMN-B & Branch & Aneuploid & 1.4 & 5 & No & 5.8 & Yes & Yes & 2 \\
16 & IPMN-B & Branch & Aneuploid & 1.4 & 13 & Yes & 3.1 & Yes & Yes \\
& $p$-Value* & 0.29 & 0.01 & 0.01 & & & & & 3 \\
\hline
\end{tabular}

IPMN-A: low-grade dysplasia; IPMN-B: medium-grade dysplasia. *Comparison between IPMN-A and IPMN-B.

three out of four patients (Table III). One patient in the IPMN-A group had an aneuploid histogram (DNA index=1.3). The DNA index and ploidy status were significantly different between the IPMN-A and IPMN-B groups $(p=001)$.

In contrast to the Sendai criteria, the ploidy index was able to differentiate between IPMN-A and IPMN-B groups, regardless of the type of IPMN (MD-IPMN vs. BD-IPMN).

Correlation between imaging criteria and DNA index. A total of 11 out of the 12 patients in the IPMN-B group both met one or more positive high-risk Sendai criteria and had aneuploid histograms with median DNA index $\geq 1.3$. One patient in this group was Sendai-negative but presented an aneuploid histogram.

In the IPMN-A group, two patients were both Sendainegative and had diploid histograms. The only patient meeting one or more positive high-risk Sendai criteria in the IPMN-A group presented a diploid histogram whereas another patient in this group presented with an aneuploid histogram but was negative for high-risk Sendai criteria (Table III).

Overall, all 11 patients who had both at least one high-risk feature according to Sendai criteria and an aneuploid histogram had an IPMN-B. The two patients who were negative for Sendai high-risk stigmata and had diploid histograms both had an IPMN-A. Out of the three patients who presented only either a Sendai worrisome feature or an aneuploid histogram, two patients had an IPMN-A and one patient had an IPMN-B (Tables III and IV).

\section{Discussion}

Most surgical centers follow the guidelines of the Sendai conference regarding the diagnosis and therapeutic treatment of IPMNs $(3,4)$. However, some authors still recommend a generous indication for pancreatic resection in patients with IPMN. Their argument is based on the assumption that a subset of even BD-IPMNs are at high risk of malignant transformation and these lessors should therefore be removed by resection (19-21). Another clinical point which is debated controversially is how to proceed in patients after pancreatic head resection for MD-IPMN with the additional manifestation of BD-IPMN in the pancreatic remnant. The question in such scenarios is always whether total duodenopancreatectomy, with all its associated operative risk and clinical consequences, or surveillance is indicated (22, 23). In any case of this kind, the risk of missing the appropriate time for resection before malignant transformation occuring during monitoring cannot be completely excluded. The Sendai 2012 guidelines are currently considered the goldstandard tool in this constellation, but are, however, limited by their low positive predictive value. Moreover, the restriction of the defined predictive criteria for malignancy in BD-IPMN is a key problem of the guidelines. As in other premalignant lesions, a precise diagnosis is the key for determining appropriate therapy. In order to avoid underdiagnosing malignant lesions and to prevent unnecessary surgical interventions on the other hand, additional objective tools for accurate sensitive prediction are urgently needed. In this context, information about the DNA status of the IPMN 
Table IV. Sensitivity, specificity and positive predictive value (PPV) for Sendai criteria and DNA index (DI).

\begin{tabular}{|c|c|c|c|c|}
\hline Discrimination by & Sensitivity & Specificity & PPV & NPV \\
\hline$\geq 2$ Positive Sendai criteria for IPMN B & $33 \%(4 / 12)$ & $75 \%(3 / 4)$ & $80 \%(4 / 5)$ & $27 \%(3 / 11)$ \\
\hline$\geq 1$ Positive Sendai criteria for IPMN B & $92 \%(11 / 12)$ & $75 \%(3 / 4)$ & $92 \%(11 / 12)$ & $75 \%(3 / 4)$ \\
\hline $\mathrm{DI} \geq 1.3$ for IPMN B & $100 \%(12 / 12)$ & $75 \%(3 / 4)$ & $92 \%(12 / 13)$ & $100 \%(3 / 3)$ \\
\hline$\geq 2$ Positive Sendai criteria + DI $\geq 1.3$ for IPMN B & $100 \%(4 / 4)$ & $100 \%(12 / 12)$ & $100 \%(4 / 4)$ & $100 \%(12 / 12)$ \\
\hline$\geq 1$ Positive Sendai criteria + DI $\geq 1.3$ for IPMN B & $92 \%(11 / 12)$ & $100 \%(4 / 4)$ & $100 \%(11 / 11)$ & $80 \%(4 / 5)$ \\
\hline Either $\geq 1$ positive Sendai criteria or DI $\geq 1.3$ for IPMN B & $86 \%(12 / 14)$ & $100 \%(2 / 2)$ & $100 \%(12 / 12)$ & $50 \%(2 / 4)$ \\
\hline
\end{tabular}

IPMN-A: Low-grade dysplasia; IPMN-B: medium-grade dysplasia.

lesions may be a helpful tool and a potential additional clinical guide for such a relevant decision. In our study, DNA ploidy, the histological IPMN subtype, as well the preoperative radiological imaging criteria, were analyzed in patients who underwent pancreatic resection for either IPMNA or IPMN-B in order to identify an additive predictive tool for the differentiation of the different IPMN subtypes. This analysis revealed a significant correlation between the histopathological finding and the DNA index: aneuploid histograms with DNA indices $\geq 1.3$ were observed in all patients with type B IPMNs. Additionally, three out of four patients with IPMN-A presented DNA indices $\leq 1.3$. In contrast to the defined criteria for malignancy, this correlation was valid for both MD-IPMN and BD-IPMN.

Interestingly, 8 out of 12 patients in the group with IPMNB did not fulfill more than one of the Sendai criteria in the preoperative imaging. These eight patients, however, all had aneuploid histograms with DNA indices $\geq 1.3$. In addition, the only patient with two or more positive high-risk Sendai criteria in the IPMN-A group presented a diploid histogram. As a relevant finding of our study, we also demonstrated that all patients in this study who were positive for at least one worrisome Sendai criterion and had an aneuploid histogram had IPMN-B, whereas all patients who had negative Sendai criteria and a diploid histogram had IPMN-A. The results of our image DNA cytometric analysis thus potentially revealed a rather sensitive additional tool for differentiation between these groups beyond the traditional Sendai criteria.

Our study is, of course, limited by the small sample size but in our view, based on the results of this study, image DNA cytometry may be considered a useful and possibly relevant diagnostic tool in addition to the established criteria for optimizing treatment decisions, especially in patients with MD-IPMN and BD-IPMN at high risk for potential malignant transformation into IPMN-C.

Early routine EUS-FNA may, therefore, be considered as a rather less-invasive important diagnostic tool in this patient population for the determination of the DNA index and thus may play an important role for individual precise decision making $(24,25)$.
In our view, especially in patients with either MD or BD IPMN with at least one positive Sendai criterion in the radiological imaging work-up, an additional EUS-FNA of the cystic lesion or fluid should be performed for DNA image cytometric analysis. According to the results of our study, an aneuploid histogram in the FNA and the presence of at least one high-risk Sendai feature may implicate an IPMN-B and thus support further clinical therapy strategy. An additional analysis of the cyst fluid with regard to KRAS as well as carcinoembryonic antigen or carbohydrate antigen 19-9 status, as described by others, may of course be added (26, 27). It should be stated however, that in our study, DNA cytometric analysis was only performed on resected specimens. Despite a reported sensitivity of FNA in other pancreatic lesions of $>76 \%$, the exact detection accuracy of FNA-based DNA-index assessment has yet to be proven for IPMN $(28,29)$.

\section{Conclusion}

As a conclusion of this study, DNA image cytometry may be considered an important additional diagnostic tool for further differentiation between IPMN-A and IPMN-B for both MDand BD-IPMN beyond the traditional Sendai criteria and may thus be helpful in guiding the monitoring process and treatment decisions in patients with MD-IPMN or BDIPMN, especially in patients with at least one positive Sendai criterion in the radiological diagnostic work-up. Further studies with greater numbers of patients are of course necessary for further evaluation of the clinical application of these data.

\section{References}

1 Hruban RH, Takaori K, Klimstra DS, Adsay NV, AlboresSaavedra J, Biankin AV, Biankin SA, Compton C, Fukushima N, Furukawa T, Goggins M, Kato Y, Klöppel G, Longnecker DS, Lüttges J, Maitra A, Offerhaus GJA, Shimizu M and Yonezawa $\mathrm{S}$ : An illustrated consensus on the classification of pancreatic intraepithelial neoplasia and intraductal papillary mucinous neoplasms. Am J Surg Pathol 28: 977-987, 2004. 
2 Kamisawa T, Fujiwara T, Tu Y, Egawa N, Tanaka T, Sakaki N, Funata $\mathrm{N}$ and Koike M: Long-term follow-up of intraductal papillary adenoma of the pancreas. J Gastroenterol 37: 868-873, 2002.

3 Tanaka M, Chari S, Adsay V, Fernandez-del Castillo C, Falconi M, Shimizu M, Yamaguchi K, Yamao K, Matsuno S and International Association of Pancreatology: International consensus guidelines for management of intraductal papillary mucinous neoplasms and mucinous cystic neoplasms of the pancreas. Pancreatol Off J Int Assoc Pancreatol IAP Al 6: 17-32, 2006.

4 Tanaka M, Fernández-del Castillo C, Adsay V, Chari S, Falconi M, Jang J-Y, Kimura W, Levy P, Pitman MB, Schmidt CM, Shimizu $\mathrm{M}$, Wolfgang $\mathrm{CL}$, Yamaguchi $\mathrm{K}$, Yamao $\mathrm{K}$ and International Association of Pancreatology: International consensus guidelines 2012 for the management of IPMN and MCN of the pancreas. Pancreatol Off J Int Assoc Pancreatol IAP Al 12: 183-197, 2012.

5 Walter TC, Steffen IG, Stelter LH, Maurer MH, Bahra M, Faber W, Klein F, Bläker H, Hamm B, Denecke T and Grieser C: Implications of imaging criteria for the management and treatment of intraductal papillary mucinous neoplasms - benign versus malignant findings. Eur Radiol 25: 1329-1338, 2015.

6 Pinto AE, Pereira T, Santos M, Branco M, Dias A, Silva GL, Ferreira MC and André S: DNA ploidy is an independent predictor of survival in breast invasive ductal carcinoma: a long-term multivariate analysis of 393 patients. Ann Surg Oncol 20: 1530-1537, 2013.

7 Flezar MS, But I, Kavalar R and Us-Krasovec M: Flow and image cytometric DNA ploidy, including 5c exceeding cells, of serous borderline malignant ovarian tumors. Correlation with clinicopathologic characteristics. Anal Quant Cytol Histol Int Acad Cytol Am Soc Cytol 25: 139-145, 2003.

8 D'Urso V, Collodoro A, Mattioli E, Giordano A and Bagella L: Cytometry and DNA ploidy: clinical uses and molecular perspective in gastric and lung cancer. J Cell Physiol 222: 532-539, 2010.

9 Abou-Rebyeh H, Borgmann V, Nagel R and Al-Abadi H: DNA ploidy is a valuable predictor for prognosis of patients with resected renal cell carcinoma. Cancer 92: 2280-2285, 2001.

10 Nagel $R$ and al Abadi $H$ : The prognostic significance of ploidy and DNA-heterogeneity in the primary diagnosis and monitoring of patients with locally advanced prostatic carcinoma. Scand J Urol Nephrol Suppl 138: 83-92, 1991.

11 Weger AR, Graf AH, Askensten U, Schwab G, Bodner E, Auer $\mathrm{G}$ and Mikuz G: Ploidy as prognostic determinant in pancreatic cancer. Lancet Lond Engl 2: 1031, 1987.

12 Porschen R, Remy U, Bevers G, Schauseil S, Hengels KJ and Borchard F: Prognostic significance of DNA ploidy in adenocarcinoma of the pancreas. A flow cytometric study of paraffin-embedded specimens. Cancer 71: 3846-3850, 1993.

13 Klein F, Bahra M, Schirmeier A, Al-Abadi H, Pratschke J, Pelzer U, Oettle H, Striefler J, Riess H and Sinn M: Prognostic significance of DNA cytometry for adjuvant therapy response in pancreatic cancer. J Surg Oncol 112: 66-71, 2015.

14 Kamphues C, Al-Abadi H, Dürr A, Al-Abadi N, Schricke D, Bova R, Müller V, Stenzinger A, Klauschen F, Seehofer D, Neuhaus P and Bahra M: DNA index as a strong prognostic factor in patients with adenocarcinoma of the pancreatic head: results of a 5-year prospective study. Pancreas 42: 807-812, 2013.

15 Kamphues C, Al-Abadi N, Dürr A, Bova R, Klauschen F, Stenzinger A, Bahra M, Al-Abadi H, Neuhaus P and Seehofer $\mathrm{D}$ : The DNA index is a strong predictive marker in intrahepatic cholangiocarcinoma: the results of a five-year prospective study. Surg Today 44: 1336-1342, 2014.
16 Hedley DW, Friedlander ML and Taylor IW: Application of DNA flow cytometry to paraffin-embedded archival material for the study of aneuploidy and its clinical significance. Cytometry 6: 327-333, 1985.

17 Chatelain R, Willms A, Biesterfeld S, Auffermann W and Böcking A: Automated Feulgen staining with a temperaturecontrolled staining machine. Anal Quant Cytol Histol Int Acad Cytol Am Soc Cytol 11: 211-217, 1989.

18 Böcking A, Giroud F and Reith A: Consensus report of the ESACP task force on standardization of diagnostic DNA image cytometry. European Society for Analytical Cellular Pathology. Anal Cell Pathol J Eur Soc Anal Cell Pathol 8: 67-74, 1995.

19 Fritz S, Klauss M, Bergmann F, Strobel O, Schneider L, Werner J, Hackert $\mathrm{T}$ and Büchler MW: Pancreatic main-duct involvement in branch-duct IPMNs: an underestimated risk. Ann Surg 260: 848-855; discussion 855-856, 2014.

20 Fritz S, Klauss M, Bergmann F, Hackert T, Hartwig W, Strobel O, Bundy BD, Büchler MW and Werner J: Small (Sendai negative) branch-duct IPMNs: not harmless. Ann Surg 256: 313-320, 2012.

21 Fritz S, Hackert $T$ and Büchler MW: Pancreatic intraductal papillary mucinous neoplasm--where is the challenge? Dig Dis Basel Switz 33: 99-105, 2015.

22 Nimptsch U, Krautz C, Weber GF, Mansky T and Grützmann R: Nationwide In-hospital Mortality Following Pancreatic Surgery in Germany is Higher than Anticipated. Ann Surg, 2016.

23 Andrén-Sandberg Å, Ansorge C and Yadav TD: Are There Indications for Total Pancreatectomy in 2016? Dig Surg 33: 329334, 2016.

24 Ridtitid W, DeWitt JM, Schmidt CM, Roch A, Stuart JS, Sherman S and Al-Haddad MA: Management of branch-duct intraductal papillary mucinous neoplasms: a large single-center study to assess predictors of malignancy and long-term outcomes. Gastrointest Endosc 84: 436-445, 2016.

25 Tanaka M: Current roles of endoscopy in the management of intraductal papillary mucinous neoplasm of the pancreas. Dig Endosc Off J Jpn Gastroenterol Endosc Soc 27: 450-457, 2015.

26 Kadayifci A, Atar M, Wang JL, Forcione DG, Casey BW, Pitman $\mathrm{MB}$ and Brugge WR: The Value of Adding GNAS Testing to Pancreatic Cyst Fluid KRAS and CEA $<m t>$ Analysis for the Diagnosis of Intraductal Papillary Mucinous Neoplasms. Dig Endosc Off J Jpn Gastroenterol Endosc Soc, 2016.

27 Moris M, Raimondo M, Woodward TA, Skinner V, Arcidiacono PG, Petrone MC, De Angelis C, Manfrè S, Fusaroli P, Asbun H, Stauffer J and Wallace MB: Diagnostic accuracy of endoscopic ultrasound-guided fine-needle aspiration cytology, carcinoembryonic antigen, and amylase in intraductal papillary mucinous neoplasm. Pancreas 45: 870-875, 2016.

28 Sur YK, Kim YC, Kim JK, Lee JH, Yoo BM and Kim YB: Comparison of ultrasound-guided core needle biopsy and endoscopic ultrasound-guided fine-needle aspiration for solid pancreatic lesions. J Ultrasound Med Off J Am Inst Ultrasound Med 34: 2163-2169, 2015.

29 Oppong KW, Dawwas MF, Charnley RM, Wadehra V, Elamin $\mathrm{K}$, White $\mathrm{S}$ and Nayar M: EUS and EUS-FNA diagnosis of suspected pancreatic cystic neoplasms: Is the sum of the parts greater than the CEA? Pancreatol Off J Int Assoc Pancreatol IAP Al 15: 531-537, 2015

Received November 20, 2016

Revised December 15, 2016 Accepted December 21, 2016 